Artículo original

(Original paper)

\title{
ESTUDIO MORFOLÓGICO DE ALEUROPLEUROCELUS ABNORMIS (QUAINTANCE) (HEMIPTERA: ALEYRODIDAE) Y NUEVOS REGISTROS DE ESPECIES DEL GÉNERO PARA MÉXICO
}

\author{
MORPHOLOGICAL STUDY OF ALEUROPLEUROCELUS ABNORMIS \\ (QUAINTANCE) (HEMIPTERA: ALEYRODIDAE) AND NEW RECORDS \\ OF SPECIES OF THE GENUS FOR MEXICO
}

\author{
VICENTE EMILIO CARAPIA-RUIZ, 1,* ÓSCAR ÁNGEL SÁNCHEZ-FLORES, ${ }^{2}$ OSWALdo \\ GARCÍA-MARTÍNEZ ${ }^{2}$ Y ANTONIO CASTILLO-GUTIÉRREZ ${ }^{1}$
}
'Universidad Autónoma del Estado de Morelos (UAEM), Escuela de Estudios Profesionales de Xalostoc (EESuX). Av. Nicolás Bravo s/n, Parque Industrial Cuautla, Xalostoc, Ayala, Morelos, México <vcarapia@hotmail.com>,<antoniocg62@hotmail.com>
${ }^{2}$ Departamento de Parasitología Agrícola, Universidad Autónoma Agraria Antonio Narro, Calzada Antonio Narro No 1923, Colonia Buenavista, 25315, Saltillo, Coahuila, México.<oscaruaaan@gmail.com>, <drogarcia@yahoo.com.mx>
* Autor para correspondencia:<vcarapia@hotmail.com>

Recibido: 31/01/2017; aceptado: 08/11/2017; publicado en línea: 16/03/2018

Editor responsable: Jesús Romero Nápoles

Carapia-Ruiz, V. E., Sánchez-Flores, O. A., García-Martínez, O. y Castillo-Gutiérrez, A. (2018) Estudio morfológico de Aleuropleurocelus abnormis (Quaintance) (Hemiptera: Aleyrodidae) y nuevos registros de especies del género para México. Acta Zoológica Mexicana (n.s), 34(1), 1-5.

RESUMEN. Aleuropleurocelus abnormis (Quaintance, 1900) es una mosca blanca de la subfamilia Aleurodinae la cual fue poco estudiada en su clasificación inicial. En este estudio se da una descripción morfológica detallada para contribuir en el actual estado y desarrollo de la taxonomía del género. Los especímenes revisados fueron de diferentes estados de México y EUA, las características morfológicas son ilustradas por medio de fotografía digital y se discuten diferencias de A. nevadiensis con otras especies de forma oval. Especímenes de $A$. abnormis se encontraron en un ejemplar de herbario en una especie de Quercus con etiqueta de colecta de Luisiana, EUA. Se reporta nuevo registro de A. hyptisemoryi para Baja California Sur y Baja California México en Hyptis emoryii y Hipis albida.

Palabras clave: moscas blancas, Aleyrodinae, Tetralicia, morfología.

\section{INRODUCCIÓN}

La mosca blanca Aleuropleurocelus abnormis (Quaintance) (Hemiptera: Aleyrodidae: Aleurodinae) fue descrita por Quaintance (1900) y clasificada en el género Aleyrodes de especímenes de diferentes localidades de Florida, EUA colectados en Quercus, Persea y Magnolia. Quaintance y Baker (1914) transfiererón a A. abnormis
Carapia-Ruiz, V. E., Sánchez-Flores, O. A., García-Martínez, O., \& Castillo-Gutiérrez, A. (2018) Morphological study of Aleuropleurocelus abnormis (Quaintance) (Hemiptera: Aleyrodidae) and new records of species of the genus for Mexico. Acta Zoológica Mexicana (n.s), 34(1), 1-5.

ABSTRACT. Aleuropleurocelus abnormis (Quaintance, 1900) is a whitefly of the subfamily Aleurodinae which was poorly studied in its initial classification, this study gives a detailed description for the current state and development of the taxonomy of the genus. The specimens used were from different states of Mexico and the USA, the morphological characteristics are illustrated by means of digital photography and differences are discussed with $A$. nevadiensis and with others not classified. A. abnormis was found in herbarium in a species of Quercus with tag Collection of Louisiana USA. A new record of $A$. hyptisemoryi for Baja California Sur and Baja California México is reported on Hyptis emoryii and Hipis albida.

Key words: whiteflies, Aleyrodinae, Tetralicia, morphology.

al género Tetraleurodes. Harrison en 1917 describe a Tetralicia en Inglaterra incluyendo sólo a la especie T. ericae (Martin, 2005); años después Baker (1937) describe a $T$. rotunda (Aleuropleurocelus rotunda) de México, que por fotografías de la descripción original parece corresponder o ser muy similar a A. abnormis, Baker aparentemente no revisó a $A$. abnormis dado que no realizó comentario ni cita a esta especie en su publicación. Sampson y Drews 
(1941) citaron a $A$. rotunda sin mencionar a A. abnormis y estudiaron otras especies de este género, en especial a las mexicanas que presentan una forma de bote muy característica. Fueron Drews y Sampson (1956) quienes establecieron el género Aleuropleurocelus para separar las especies de California, EUA del género Tetralicia, que incluyen las especie europeas de pupas de mosca blanca con el margen doblado hacia la superficie ventral. Martin (2005) hace la transferencia de Tetraleurodes abnormis a Aleuropleurocelus abnormis y poco tiempo después Evans (2007) registró a A. abnormis para Florida, Arizona y Texas en EUA y México, además de intercepciones de Colombia, República Dominicana, El Salvador y Perú. Por su parte Sanchez-Flores et al. (2017) reportaron para Tepeaca, Puebla, México a A. abnormis.

Debido a que la descripción por Quaintance de $A$. $a b$ normis fue muy simple y sin detalles morfológicos útiles para identificación y/o separación de otras especies similares. Se realizó el siguiente estudio con la finalidad de describir de forma objetiva y con fotografía digital en microscopio compuesto; por otro lado se brindan nuevos registros para México y EUA de la especie Aleuropleurocelus hyptisemoryi.

\section{MATERIALES Y MÉTODOS}

Se hicieron colectas de pupas de moscas blancas en el mes de diciembre de 2015 a julio de 2016 en los municipios de Tepeaca y los Reyes de Juárez del estado de Puebla. Para este propósito, se observaron hojas de diferentes cultivos, maleza, arbustos y árboles presentes en parcelas, patios y traspatios de casas, calles y en cerros ubicados al norte de esta comunidad; también se colectaron especímenes en las muestras del herbario de esta misma universidad (ANSM), además se hicieron colectas principalmente en aguacate y en diferentes especies del genero Quercus en el sur de Coahuila. Con tijeras se cortaron pedazos de las hojas donde estaban las ninfas y se colocaron dentro de cajas Petri que se sellaron en sus cantos con Kleen Pack ${ }^{\circledR}$, y se les colocaba una etiqueta donde se anotaban los datos de colecta. Las cajas Petri se transportaron al Laboratorio de Taxonomía de Insectos y Ácaros del Departamento de Parasitología Agrícola-Saltillo de la Universidad Autónoma Agraria Antonio Narro. Las pupas se montaron en porta y cubreobjetos, siguiendo la metodología de Martin (2004) con algunas modificaciones. También se estudiaron aproximadamente 100 especímenes de la colección a resguardo del primer autor.
Para observar las preparaciones se utilizó un microscopio compuesto marca Olimpus ${ }^{\circledR}$ considerando 40, 100, 400 y 1000 X. La identificación de las especies se realizó utilizando descripciones y claves de Sampson y Drews (1941) y Martin (2005). Las plantas hospederas fueron identificadas por el Dr. José Ángel Villarreal Quintanilla (ANSM).

\section{RESULTADOS}

Redescripción de Aleuropleurocelus abnormis (Quaintance). Forma general. Pupas de color negro, que por lo general se localizan en el envés de las hojas, se distingue un pequeño halo de cera alrededor del cuerpo, que se desprende fácilmente de las pupas al separarse de las hojas. Especímenes en portaobjetos. Cuerpo oval con $670 \mu \mathrm{m}$ de largo por $470 \mu \mathrm{m}$ de ancho (Fig. 1). Margen deflejado aproximadamente 0.6 veces como el ancho del cuerpo. Margen. Submargen tuberculado, los gránulos arreglados de forma irregular, margen aparente (pliegue submarginal) débilmente dentado, margen verdadero con dientes cuadrangulares (Fig. 8); submargen esculpido con tubérculos escasos cerca de la línea submarginal y más abundantes hacia el margen con una especie de ramas transversas que son más abundantes en los dos tercios próximos al margen (Fig. 2). Cefálotorax. Ojos ovales de $23 \mu \mathrm{m}$ de largo y $14 \mu \mathrm{m}$ de ancho, con una estructura oval dividida longitudinalmente en dos áreas cerca del pliegue submarginal y la sutura longitudinal de la muda, ligeramente posterior a esta estructura se presentan tres pares de depresiones bien definidas con dientes pequeños de 14-18 $\mu \mathrm{m}$ de largo y 8-10 $\mu \mathrm{m}$ de ancho y un par de setas cefálicas de $17 \mu \mathrm{m}$ de largo (Fig. 3-5). Zona media torácica con dos pares de setas, las mesotóracicas de $21 \mu \mathrm{m}$ de largo y metátorcicas de $19 \mu \mathrm{m}$ de largo, depresiones con tubérculos pequeños alrededor de éstas. Sutura longitudinal de la muda de $370 \mu \mathrm{m}$ de largo con una banda de tubérculos de $7 \mu \mathrm{m}$ de largo en cada lado, aparentando una forma de cremallera (Fig. 5), sutura transversal de la muda $450 \mu \mathrm{m}$ de largo, sin tubérculos, se extiende en forma curva y termina un poco antes de llegar al margen aparente. Abdomen. Segmentos abdominales I-VIII claramente visibles en la parte media, con longitud aproximada para el segmento I de $34.2 \mu \mathrm{m}$, segmento II $27.8 \mu \mathrm{m}$, segmento III $29.2 \mu \mathrm{m}$, segmento IV $30.6 \mu \mathrm{m}$, segmento V $37.7 \mu \mathrm{m}$, segmento VI $32.8 \mu \mathrm{m}$, segmento VII $29.3 \mu \mathrm{m}$, y segmento VII $49.3 \mu \mathrm{m}$. Depresiones abdominales presentes y bien definidas, con pequeños tubérculos con las siguientes dimensiones: seg- 

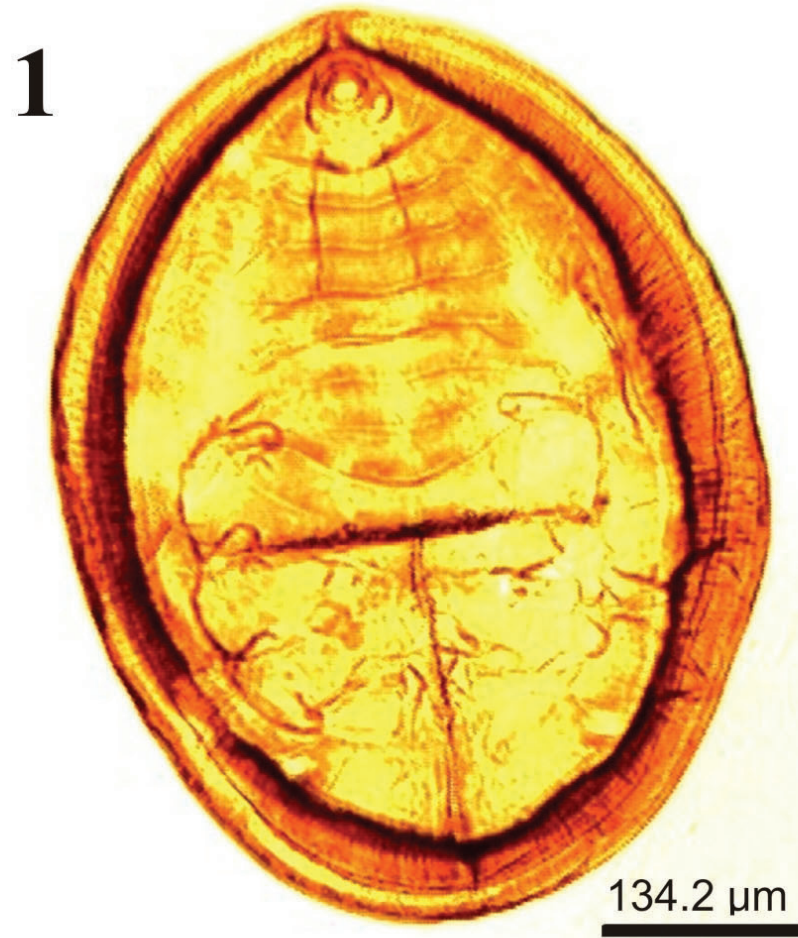
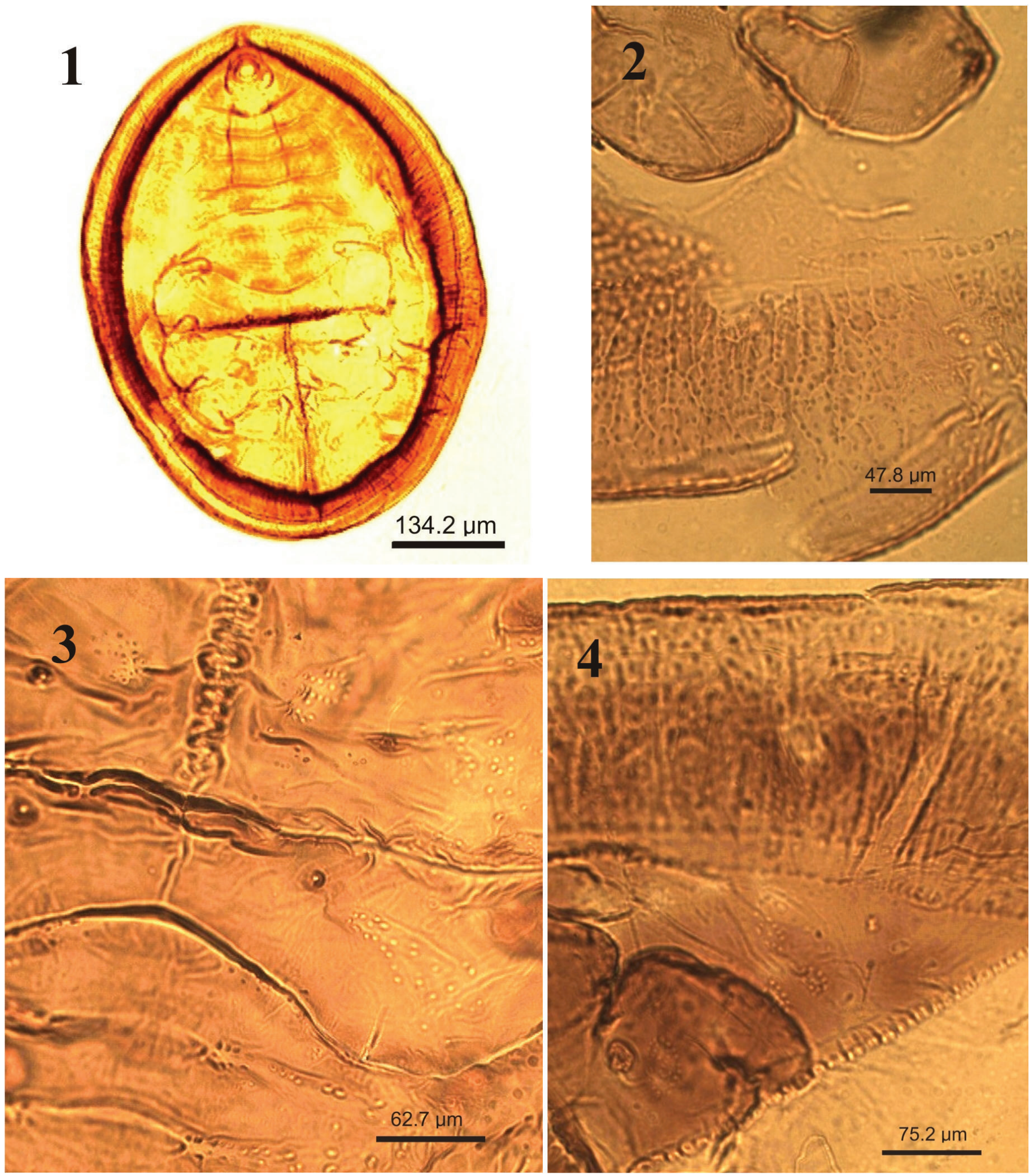

Figuras 1-4. Aleuropleurocelus abnormis (Quaintance). 1) Pupa, 2) Banda de tubérculos y ramificaciones en el submargen, 3) Cefálotorax y primeros segmentos abdominales, 4) Depresiones cefálotoracicas. 

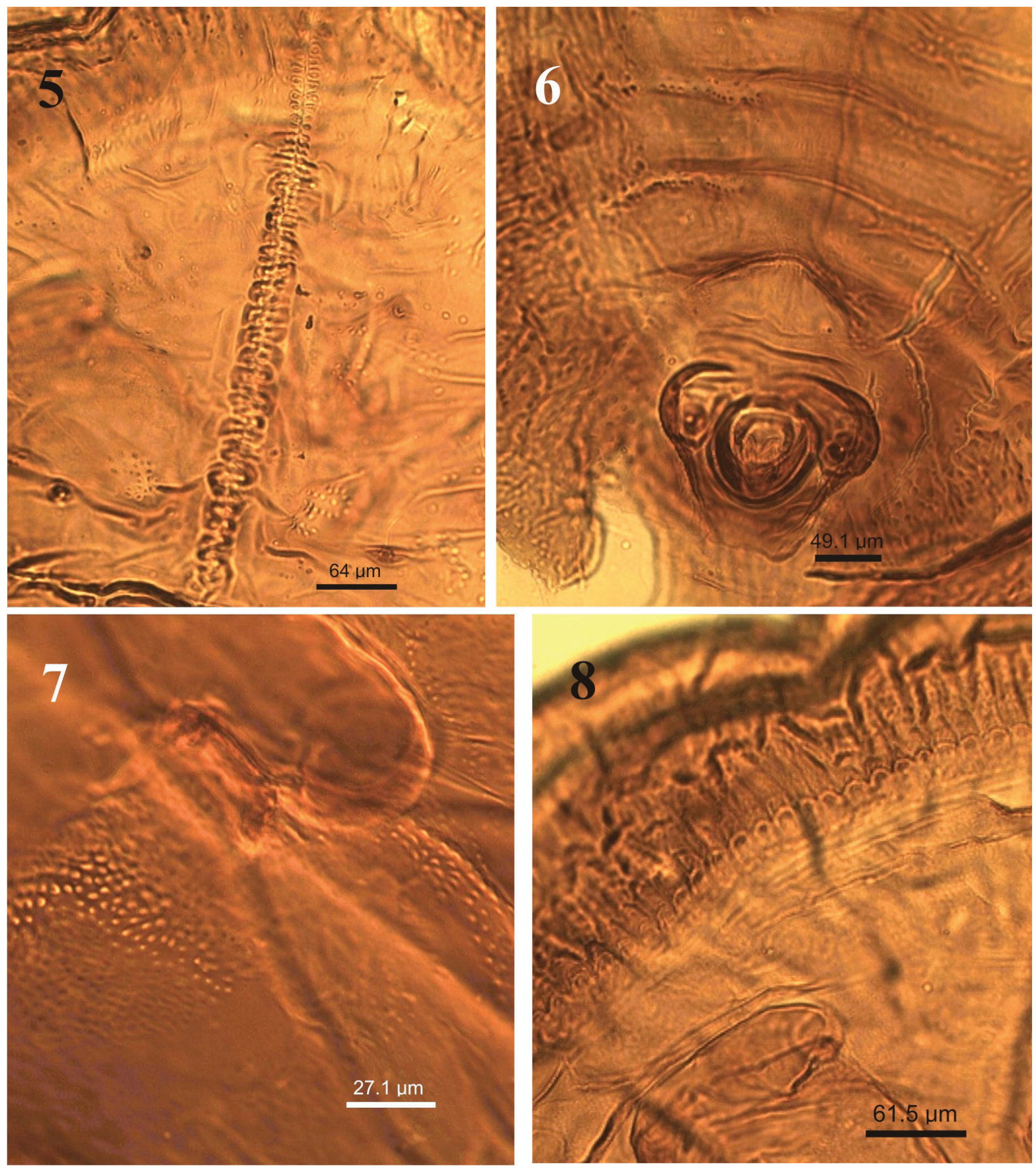

Figuras 5-8. Aleuropleurocelus abnormis (Quaintance). 5) Sutura longitudinal o media de la muda, 6) Orificio vasiforme y parte media del abdomen, 7) Espínulas en la base de las patas, 8) Dientes marginales. 
mento I $7 \mu \mathrm{m}$ de largo y $17.5 \mu \mathrm{m}$ de ancho, segmento II 9 $\mu \mathrm{m}$ de largo y $24.9 \mu \mathrm{m}$ de ancho, segmento III $8.1 \mu \mathrm{m}$ de largo y $18.9 \mu \mathrm{m}$ de ancho, segmento IV $7.3 \mu \mathrm{m}$ de largo y $18.6 \mu \mathrm{m}$ de ancho, segmento V $7.7 \mu \mathrm{m}$ de largo y $18.3 \mu \mathrm{m}$ de ancho, segmento VI $7 \mu \mathrm{m}$ de largo y $18.6 \mu \mathrm{m}$ de ancho; las líneas de tubérculos entre los segmentos abdominales se extienden desde las depresiones medias hacia el margen aparente, parte media de los segmentos abdominales casi liso (Fig. 3). Orificio vaciforme. Semiovoide, $38 \mu \mathrm{m}$ largo por $40 \mu \mathrm{m}$ en la parte más ancha; opérculo $35 \mu \mathrm{m}$ largo por $33 \mu \mathrm{m}$ de ancho, cubre completamente la língula y a la mayoría del orificio vasiforme, anillo de orificio vasiforme de $40 \mu \mathrm{m}$ de ancho; orificio vasiforme y setas abdominales VIII con cierta elevación, claramente diferenciada del área adyacente en especímenes montados. Distancia del margen posterior del orificio vasiforme al margen aparente de $44.7 \mu \mathrm{m}$, protuberancia caudal ausente o reducida en gran medida (Fig. 6). Vientre. Antenas se extienden posteriormente al par de las patas protóracicas de $90 \mu \mathrm{m}$ de largo y $37 \mu \mathrm{m}$ de ancho, patas mesotóracicas de $101 \mu \mathrm{m}$ de largo y $48 \mu \mathrm{m}$ de ancho en el segmento basal, patas metatóracicas de $121 \mu \mathrm{m}$ de largo y $55 \mu \mathrm{m}$ de ancho, base de las patas con una banda ancha irregular de espínulas de $6 \mu \mathrm{m}$ de largo y $2.5 \mu \mathrm{m}$ de ancho (Fig. 7), cutícula torácica aparentemente lisa en el resto de la parte ventral, un par de sacos adhesivos cerca de la base del primer par de patas, cutícula abdominal lisa (Fig. 3-5). Quetotaxia. Con un par de setas cefálicas, un par mesotóracico, un par metatóracico y un par en el segmento abdominal VIII, cada una derivada de una base tuberculada; en el margen anterior-lateral del orificio vasiforme con dos setas caudales presentes de $66.2 \mu \mathrm{m}$ de largo.

Registro de especies de Aleuropleurocelus. Además de las localidades antes indicadas, $A$. abnormis se registra de una muestra de Quercus stellata Wangenh depositado en el herbario de la Universidad Autónoma Agraria Antonio Narro (ANSM) el cual tiene etiqueta de colecta del estado de Luisiana, EUA. Para la especie A. hyptisemoryi se encontró en muestras del herbario de la misma universidad (ANSM) cuyas etiquetas indicaban los lugares de colecta en Baja California Sur y Baja California, México de los hospederos Hyptis emoryi Torr., y Hipis albida Kunth (Lamiaceae).

\section{DISCUSIÓN}

En el género Aleuropleurocelus se presenta un grupo de especies, muy común en México, que se caracterizan por presentar una forma oval muy característica; dentro de este grupo de especies A. abnormis (Quaintance) se distingue de otras por presentar tres pares de depresiones cefálicas y un par torácico que tiene forma ancha e irregular, las depresiones abdominales en la parte media dorsal también son características en esta especie, así como también una banda ramificada y tuberculada en el submargen, que no presente en la especie $A$. nevandiesis ni en alguna otra especie.

AGRADECIMIENTOS. Al Dr. José Ángel Villarreal Quintanilla por la identificación de plantas y facilidades de trabajo en el herbario (ANSM). A Martin J. H. por los artículos proporcionados y por comentarios sobre esta especie.

\section{LITERATURA CITADA}

Baker, J. M. (1937) Notes on some Mexican Aleyrodidae. Anales del Instituto de Biología de la Universidad Nacional Autónoma de México, 8, 599-629.

Drews, E. A. \& Sampson, W. W. (1956) Tetralicia and a new related genus Aleuropleurocelus (Homoptera: Aleyrodidae). Annals of the Entomological Society of America, 49, 280-283.

Evans, G. A. (2007) The whiteflies (Hemiptera: Aleyrodidae of the world and their host plants and enemies. USDA/Animal Plant Health Inspection Service (APHIS), Version 070606, 11 June 2007, pp. 708. [electronic catalogue available at: http://ebookbrowse.com/ev/evans-aleyrodidae?pdf $=$ on]

Martin, J. H. (2004) Whiteflies of Belize (Hemiptera: Aleyrodidae) Part 1-Introdution and account of the subfamily Aleurodicinae Quaintance \& Baker. Zootaxa, 681, 1-119.

Martin, J. H. (2005) Whiteflies of Belize (Hemiptera: Aleyrodidae) Part 2- a review of the subfamily of the subfamily Aleyrodinae Quaintance \& Baker. Zootaxa, 1098, 1-116.

Quaintance, A. L. (1900) Contribution towards a monograph of the American Aleurodidae. Technical Series, United States Department of Agriculture Bureau of entomology, 8, 9-62.

Quaintance, A. L. \& Baker, A. C. (1914) Classification of the Aleyrodidae. Part II. U.S.D.A. Bur. Ent. Tech. Ser., No. 27, 95-109.

Sampson, W. W. \& Drews, E. A. (1941) Fauna Mexicana IV. A review of the Aleyrodidae of México. Anales de la Escuela Nacional de Ciencias Biológicas, 2, 143-189.

Sánchez-Flores, A., Carapia-Ruiz, V. E., García-Martínez, O., \& Villareal-Quintanilla, J. A. (2017) Moscas Blancas y sus Plantas Hospederas en Tepeaca, Puebla, México. Southwestern Entomologist, 42(2), 605-608. 